\title{
PHÁT TRIỂN CÁC MODULE HỖ TRỢ ÚNG DỤNG HỆ THỐNG TÍCH HỢP LIDAR-MÁY ẢNH SỐ TRONG THÀNH LẬP MÔ HİNH SỐ ĐỘ CAO VÀ BÌNH ĐỒ TRỤC ẢNH
}

\author{
PGS. TS. NGUYẼ̃N TRƯỜNG XUÂN(1), TS. CÁP XUÂN TÚ(2), KS. MAI VĂN SỸ ${ }^{(3)}$ \\ (1) Trường Đại học Mỏ-Địa chất; \\ (2) Tổng Công ty Tài nguyên và Môi trường Việt Nam; \\ (3) Công ty Cổ phần Tư vấn thiết kế công trình xây dựng Hải Phòng
}

\section{Tóm tắt:}

Bài báo này giới thiệu một số Module được xây dựng để hỗ trợ ứng dụng hệ thống tích hợp LiDAR - Máy ảnh số trong thiết kế kỹ thuật, xử lý thông tin để thành lập mô hình số độ cao và bình đồ trực ảnh ở nước ta.

\section{1. Đặt vấn đề}

ông nghệ LiDAR - Light Detection And

Ranging là công nghệ tiên tiến hàng

đầu trong hệ thống các công nghệ thu thập dữ liệu không gian trên thế giới. Công nghệ này đã mở ra một kỷ nguyên mới trong lĩnh vực Trắc địa - Bản đồ, nó cho phép thu nhận, hiển thị độ cao chi tiết bề mặt địa hình và tạo ảnh cường độ xám nhanh, chính xác. Hệ thống LiDAR tích hợp với máy chụp ảnh số, ngoài tạo mô hình số độ cao, còn tạo ra bình đồ trực ảnh, ảnh phối cảnh 3D và các sản phục phục vụ đa ngành khác.

Khi triển khai áp dụng công nghệ LiDAR để thu thập và xây dựng cơ sở dữ liệu không gian một khu vực sẽ phải thực hiện theo 3 giai đoạn:

Giai đoạn 1: Nghiên cứu nhiệm vụ, ước tính thông số và thiết kế kỹ thuật chung;

Giai đoạn 2: Thiết kế bay, thực hiện bay quét và xử lý dữ liệu;

Giai đoạn 3: Chuyển giao thành quả cho khâu sản xuất tiếp sau;

Phần mềm chính hãng chủ yếu thực hiện các nhiệm vụ trong giai đoạn 2 , trong thực tế sản xuất, nhu cầu giải quyết vấn đề kỹ thuật phát sinh trong giai đoạn 1,3 và cả trong giai đoạn 2 lại rất đa dạng. Khi bắt đầu triển khai dự án, rất cần phải ước tính mật độ điểm quét laser, độ phân giải mặt đất của ảnh phù hợp theo yêu cầu nhiệm vụ đặt ra; ước tính các thông số tối ưu về độ cao, vận tốc, tần số phát xung, thông số máy ảnh; quản lý dữ liệu; ước tính khối lượng bay quét và chụp ảnh (giai đoạn 1); tính chuyển số liệu cho phù hợp các ứng dụng của khâu sản xuất tiếp sau (giai đoạn 3); trợ giúp, mở rộng chuẩn hoá DEM trong phần xử lý dữ liệu (giai đoạn 2)... Với các nhu cầu kỹ thuật thực tiễn nêu trên, chúng ta cần phải xây dựng các phần mềm hỗ trợ khi ứng dụng phù hợp với điều kiện sản xuất của Việt Nam. trơ'

\section{Giới thiệu các Module phần mềm hỗ}

\subsection{Modul hỗ trợ tính các thông số thiết kế}

* Modul "Ước tính mật độ điểm quét laser và độ phân giải ảnh" (Xem hình 1)

Mật độ điểm quét laser khi ước tính trong thiết kế kỹ thuật phụ thuộc chủ yếu vào yêu cầu độ chính xác của DEM và có tham chiếu tới mật độ điểm DSM phục vụ nắn trực ảnh. Ước tính mật độ điểm quét laser được tính bằng công thức gần đúng của Arckermann. 
Độ phân giải mặt đất của ảnh gốc được ước tính theo yêu cầu của tỷ lệ bản đồ cần thành lập. Sự ước tính các thông số này mang tính tham khảo, trong thực tế phải linh hoạt lựa chọn cho phù hợp với yêu cầu kỹ thuật và các điều kiện khác của khu đo. Modul được viết bằng ngôn ngữ Visual Basic.

* Modul "Ước tính và lựa chọn các thông số bay quét laser"

Công tác tính toán và lựa chọn các thông số bay quét phù hợp trong hệ thống LiDAR thực tế là giải mối quan hệ giữa độ cao bay, vận tốc bay, tần số phát xung, tần số quét và mật độ điểm quét laser, khoảng cách cân đối giữa hai điểm quét laser gần kề theo hướng bay và vuông góc với hướng bay.

Modul này sẽ tính để lựa chọn các thông số bay quét laser tương ứng với nhau theo các bước giá trị thay đổi của tần số phát xung, tần số quét khi nhập thông số vận tốc, độ cao bay và góc mở quét. Khi lựa chọn thông số, phải xuất phát từ mật độ điểm quét laser và ưu tiên chọn độ cao bay tối đa.

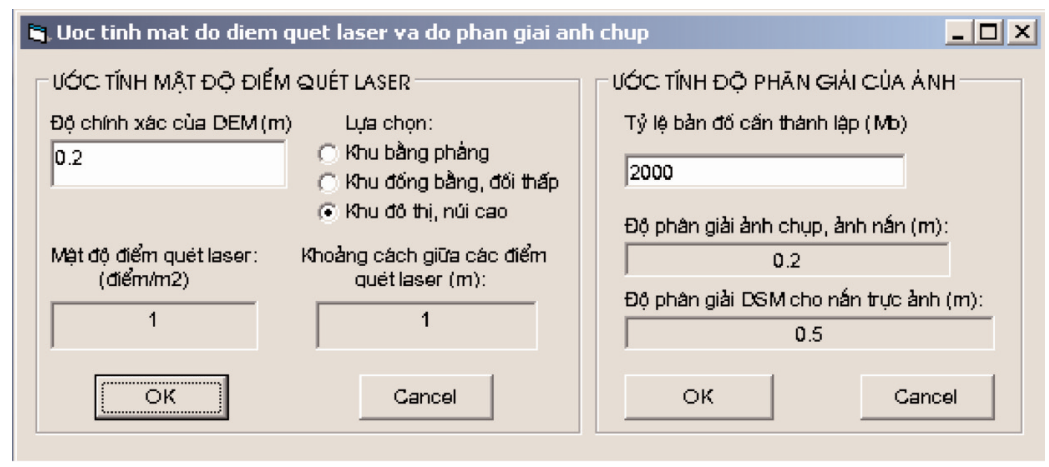

Hình 1: Giao diện ước tính mật độ điểm quét laser và độ phân giải ảnh

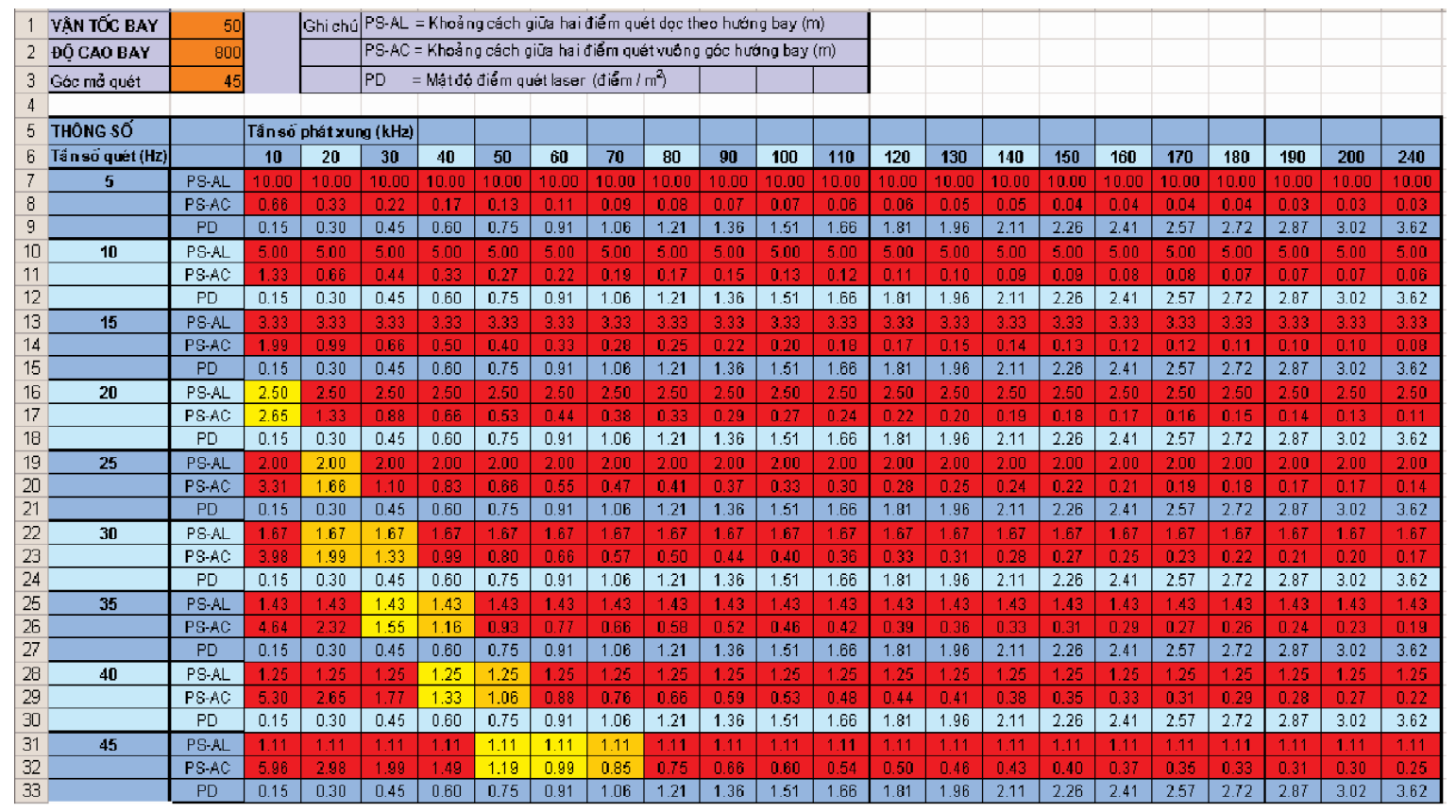

Hình 2: Giao diện ước tính và lựa chọn các thông số bay quét laser 


\begin{tabular}{|c|c|c|c|}
\hline XÍ NGHIỆP BAY CHỤP VÀ ĐO VË ẢNH - ThV_ & & & \\
\hline \multicolumn{4}{|l|}{ TÍNH THÓNG SO̊́ BAY QUÉT LIDAR - H5b } \\
\hline Hộ thống LIDAR & Harrier 56 & & \\
\hline Thông só & Gla tr! & ๒on v! & Dån glàl \\
\hline \multirow[t]{2}{*}{ Nhâp góc quêt } & 45 & degrees (dô) & Ok \\
\hline & 0.79 & $\mathrm{rad}$ & Dữ liẹu tính dược \\
\hline \multirow[t]{3}{*}{ Nhầp vần tớc bay } & 50 & $\mathrm{~m} / \mathrm{s}$ & OK \\
\hline & 180 & $\mathrm{~km} / \mathrm{h}$ & Dữ liẹu tính dược \\
\hline & 97 & $\mathrm{kts}$ & Dứ liệ tính đaực \\
\hline Nhâp dộ cao bay & 800 & $m$ & OK \\
\hline Nhâp tần số quèt & 70 & $\mathrm{~Hz}$ & Ok \\
\hline \multirow[t]{2}{*}{ Whâp tốc dộ phàt xung } & 120 & $\mathrm{kHz}$ & OK \\
\hline & 1,189 & $m$ & Chiè̀u cao bay tói da \\
\hline tồ rộng dường bay & 663 & $m$ & Dữ liệ tính dıực \\
\hline Hlộu suắt bay quèt & 119 & $\mathrm{~km}^{2} / \mathrm{gic}$ & Khöng tinh thdi gian quay vong \\
\hline Khoàng cach glü̉s hal dlểm quèt lasor gản kẻ & 0.71 & m (doc hutúng bay) & Dữ liẹu tính dưṭ̛c \\
\hline Khoàng càch glửa hal dlểm quèt laser gản kể & 0.77 & m (ngang hưung bay) & Dữ liẹu tính đạực \\
\hline mật dộ dal̂̉m quét laser & 1.81 & diểm $/ \mathrm{m}^{2}$ & Dứ lièu tính đưtuc \\
\hline Đường kinh chùm tla quèt o d măt dất & 0.40 & $m$ & Dữ liệu tính dıực \\
\hline Ghl chù & Chi nhàp d & Ự lièu trong ó có mầu c & yreen \\
\hline
\end{tabular}

\section{Hình 3: Giao diện tính các thông số bay quét laser}

Tần số phát xung phải lựa chọn tương ứng với độ cao bay và chỉ nên chọn mật độ điểm quét có tỷ lệ sai lệch khoảng cách giữa chiều dọc và ngang không quá 25\% (mầu vàng). Modul được viết trực tiếp trên Microsoft Excel.

\section{* Modul "Tính thông số bay quét LiDAR - H56"}

Để giúp công tác thiết kế bay chụp và chọn chính xác thông số cài đặt trong hệ thống LiDAR, Modul sẽ tính các thông số cụ̣ thể về độ rộng đường bay, chiều cao bay tối đa, hiệu suất bay quét, khoảng cách giữa hai điểm quét laser gần kề theo hướng bay và vuông góc với hướng bay và mật độ điểm quét laser khi nhập các thông số góc quét, vận tốc bay, độ cao bay, tần số quét, tần số phát xung. Modul được viết trực tiếp trên Microsoft Excel. Từ các bảng tính này, người thiết kế sẽ lựa chọn các thông số tối ưu để bay quét và cài đặt cho hệ thống LiDAR khi bay.

\section{* Modul "Tính các thông số bay chụp ảnh"}

Khi thiết kế và ước tính các thông số cho máy ảnh thì thông số quan trọng nhất là thời gian tối thiểu cho một lần chụp. Độ cao bay, vận tốc bay, độ phủ dọc, khả năng thu nhận dữ liệu chụp của hệ thống là các tham số liên quan trực tiếp đến thời gian tối thiểu cho một lần chụp. (Xem hình 4)

Máy ảnh hàng không chuyên dụng cỡ trung bình, thường đi tích hợp với hệ thống LiDAR là Rollei AIC P45. Modul sẽ ước tính các thông số như thời gian một lần chụp ở các độ cao bay, độ phủ dọc, vận tốc bay khác nhau; kích thước thực địa ngang và 


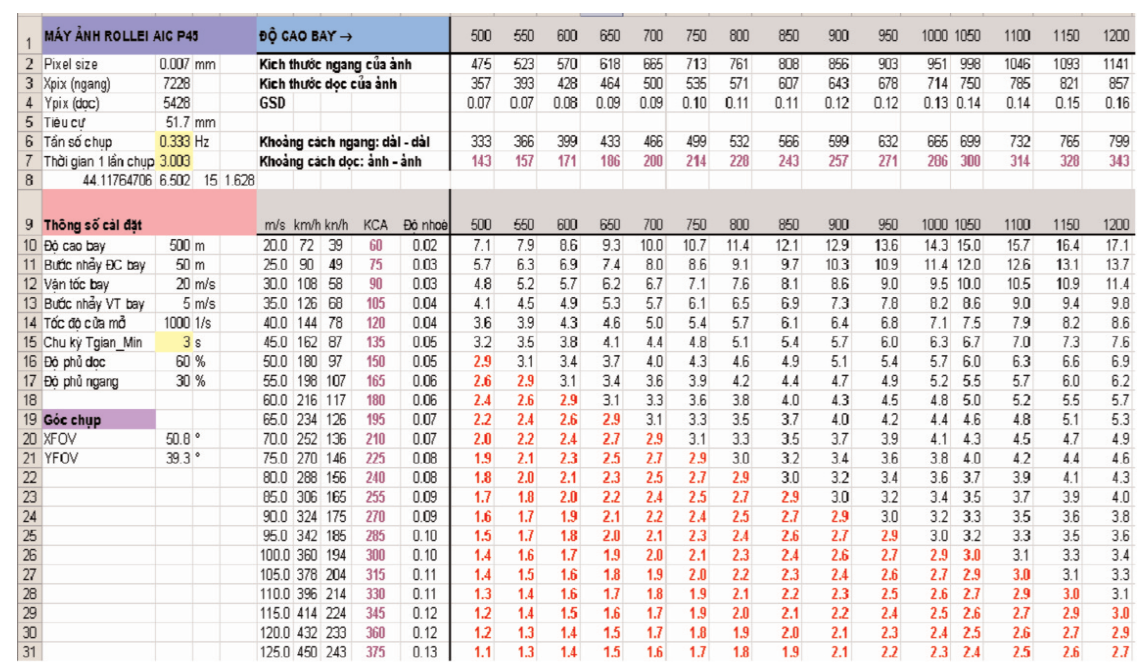

Hình 4: Giao diện tính các thông số bay chụp ảnh

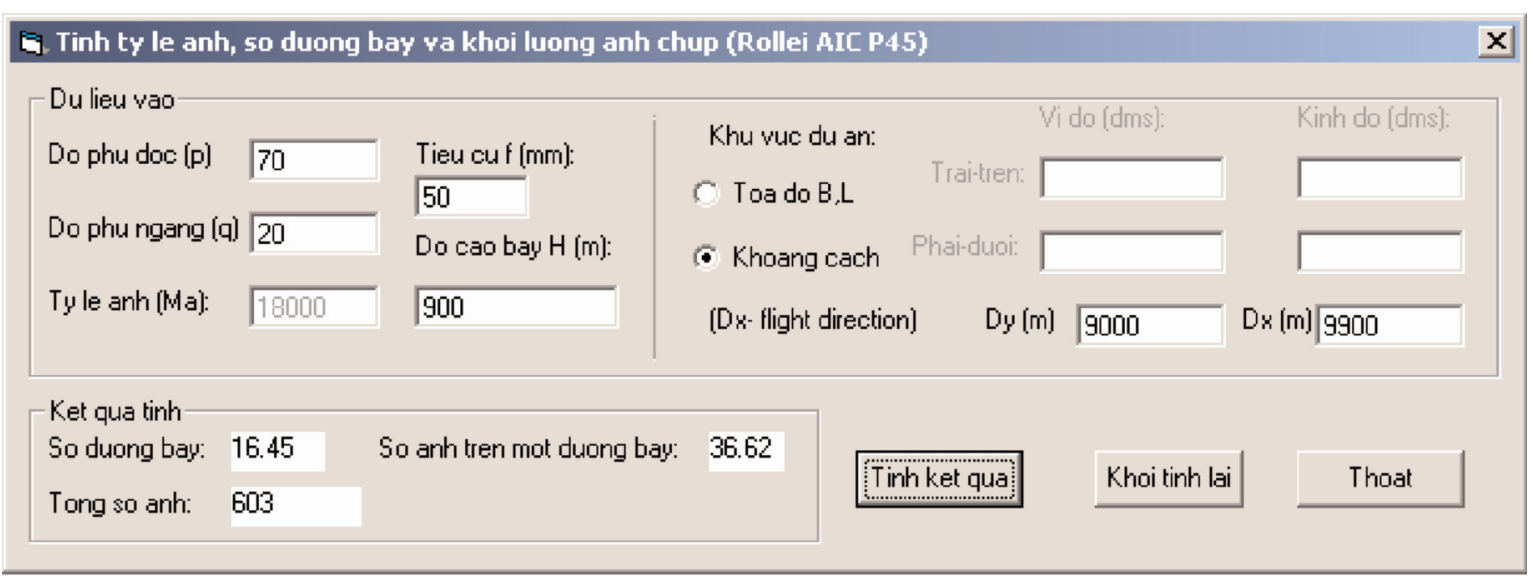

Hình 5: Giao diện tính tỷ lệ ảnh số đường bay, tổng số ảnh chụp của dụ án

dọc của ảnh; khoảng cách giữa các ảnh và giữa các dải và độ nhoè của ảnh. Các số mầu đỏ là chỉ số thời gian nhỏ hơn mức tối thiểu cho một lần chụp. Do vậy, chỉ được phép chọn độ cao bay, vận tốc và độ phủ tương ứng với thời gian chụp.

\section{* Modul "Ước tính khối lượng ảnh"}

Dùng ước tính tỷ lệ ảnh, số đường bay và tổng số ảnh chụp, tương ứng với độ phủ dọc, độ phủ ngang, tiêu cự máy ảnh, độ cao bay trong dự án. Modul được viết bằng ngôn ngữ Visual Basic.

\subsection{Modul phần mềm hỗ trọ̣ xử lý dữ liệu}

File dữ liệu LiDAR trước và sau xử lý có một đặc thù là kích thước rất lớn. Cách thức quản lý, định dạng, tổ chức file dữ liệu của sản phẩm ra đối với mỗi phần mềm xử lý LiDAR là khác nhau. Hiện nay, hầu hết các cơ sở sản xuất lớn trong nước đang biên tập bản đồ và làm dữ liệu nền thông tin địa lý đều dùng bộ Mapping office (MicroStation, IGeovec, IrasC, IrasB), MGE, trạm đo vẽ ảnh số của INTERGRAPH hay bộ ArcGIS của ESRI. Trong quá trình ứng 
dụng sản phẩm của LiDAR (hệ thống LiDAR - Harrier56) cho sản xuất đã gặp một số khó khăn do định dạng và tổ chức của dữ liệu LiDAR.

Để thuận tiện cho ứng dụng sản phẩm LiDAR phục vụ sản xuất trong điều kiện của Việt Nam, Modul phần mềm "Tính chuyển các thông số và dữ liệu LiDAR" có một số module giúp thực hiện các chuyển đổi dữ liệu phù hợp với yêu cầu kỹ thuật của các phần mềm hiện đang sản xuất trong nước. Phần mềm được viết bằng ngôn ngữ Visual Basic.

\subsection{Modul phần mềm tạo thư mục} quản lý dữ liệu

Dũ liệu gốc trong bay chụp ảnh và quét LiDAR có nhiều chủng loại và kích thước rất lớn. Các file dữ liệu quét laser, GPS/IMU, GPS mặt đất, file ảnh gốc... được thu nhận theo ca bay trong từng ngày, từng khu đo. Dữ liệu trung gian trong quá trình xử lý cũng có rất nhiều chủng loại như point_cloud, dsm_adj, dsm_fe,dsm_le, dsm_rgbi, develop_sdc, intensity, coverage_dsm, proc_laser, profiles, ảnh phát triển, sbet_file.... Trong quá trình xử lý, phải

\begin{tabular}{|c|c|c|}
\hline \multicolumn{2}{|c|}{ E- Tinh chuyen thong so ya du lieu LiDar } & $\mathbf{x}$ \\
\hline \multirow[t]{13}{*}{ File } & Tools Help & \\
\hline & 1. Chuyen toa do DEM sang mui chieu khac & \\
\hline & 2. Chia DEM theo manh Ban do & \\
\hline & 3. Chia DEM theo tile & \\
\hline & 4. Tinh chuyen EO tu WGS84 sang XVh (WN2000) & \\
\hline & 5. Tinh chuyen EO tu WGS84 sang XYH (Dia tam WN2000) & \\
\hline & 6. Tinh chuyen toa do *.TOR sang Raster (Arc GIS) & \\
\hline & 7. Tinh chuyen toa do *. TOR sang XYh & \\
\hline & 8. Tinh chuyen toa do *. TOR sang Fet (Arc GIS) & \\
\hline & 9. Tinh chuyen toa do *. TOR sang Bil (Arc GIS) & \\
\hline & 10. Tinh chuyen toa do XYh (Coo.*) sang ASCII (Arc GIS) & \\
\hline & 11. Chuven doi format ENh sang nXYh & \\
\hline & 12. Chuyen doi format $n X Y h$ sang ENh & \\
\hline
\end{tabular}

Hình 6: Giao diện tính chuyển thông số và dữ liệu LiDAR

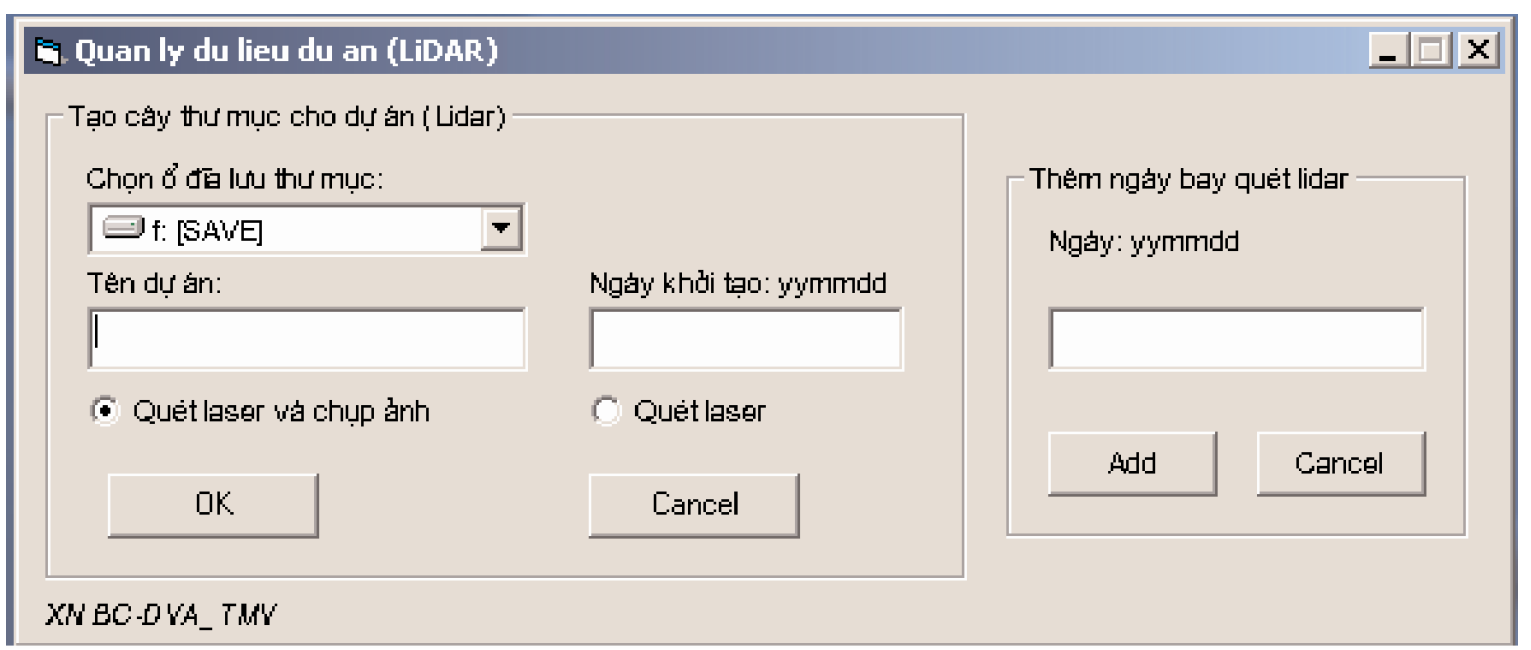

Hình 7: Giao diện quản lý dũ̃ liệu 
thường xuyên lấy dữ liệu của bước này để thực hiện bước xử lý sau. Nếu không có sự quản lý tốt, người thao tác xử lý sẽ rất dễ bị nhầm lẫn, quá trình tìm kiếm, tạo đường link giữa các máy cũng hay sai sót, nhất là cùng một thời điểm có nhiều ngày bay quét, nhiều khu đo cùng thi công và xử lý.

Xuất phát từ nhu cầu thực tế sản xuất và để quản lý tốt dữ liệu gốc, dữ liệu đã xử lý, thống nhất tên gọi thư mục, tên và phân loại dữ liệu, Modul phần mềm "Quản lý dữ liệu dự án (LiDAR)" sẽ khắc phục các vấn đề nan giải trên. Modul được viết bằng ngôn ngữ Visual Basic.

Các dữ liệu gốc và xử lý trung gian quản lý theo từng boot (ca bay quét - một ngày có thể có hơn một boot). Các dữ liệu xử lý cuối cùng đã kiểm tra (đề giao nộp) được quản lý trong Project_của khu đo.

\subsection{Một số các macro dùng trong chuẩn hoá DEM/DTM}

Khi thành lập DTM cho khu vực phức tạp (khu đô thị và vùng ven), dữ liệu LiDAR sau khi lọc tự động, kiểm tra và xử lý trên phần mềm của LiDAR tạo mô hình số địa hình, có thể vẫn còn sót hoặc chưa chuẩn xác. Thông thường là dùng bình đồ ảnh số (mầu) mở trùng khớp với DTM để kiểm tra độ hợp lý và phát hiện lỗi của DTM. Đây là công tác chuẩn hoá lại DTM.
Do số lượng khoá xử lý LiDAR rất hạn chế và khi khối lượng dự án nhiều, công tác chuẩn hoá DTM sẽ đòi hỏi khá nhiều thời gian, công sức. Để nhiều người cùng tham gia chuẩn hoá DTM, giải phóng nhanh sản phẩm, có thể sử dụng một số Macro viết hỗ trợ để chuẩn hoá DTM dựa trên phần mềm phổ thông MicroStation và ArcMap. Các macro này được viết bằng ngôn ngữ Visual basic trong môi trường Microstation.

Kết quả cuối cùng quá trình chuẩn hoá sẽ có hai file DTM theo ô lưới (grid): một file ở dạng text, một file ở dạng raster. Các file DTM dạng text có kích thước file lớn, nhưng có thể dùng cho mọi phần mềm; file dạng raster có kích thước file rất nhỏ và chỉ dùng cho các phần mềm GIS.

* Macro coo2text.ba: Do số lượng điểm trên file DTM rất lớn (mảnh 1/2000 là $70 \mathrm{mb}$ ), phần mềm MicroStation không đọc được, nên các file cần chia nhỏ ra thành 4 file để đọc và thao tác thuận lợi.

* Macro buldozer.ba: Chương trình sẽ chạy lần lượt chuẩn hoá điểm DTM trong 4 file DGN dựa vào file số hoá đã gán 3D trên *.dgn. (Xem hình 9)

* Macro dgn2txt.ba: Theo quy định, các sản phẩm DTM, bình đồ ảnh đều đóng gói và quản lý theo mảnh bản đồ. Macro dgn2txt.ba chạy trên môi trường của

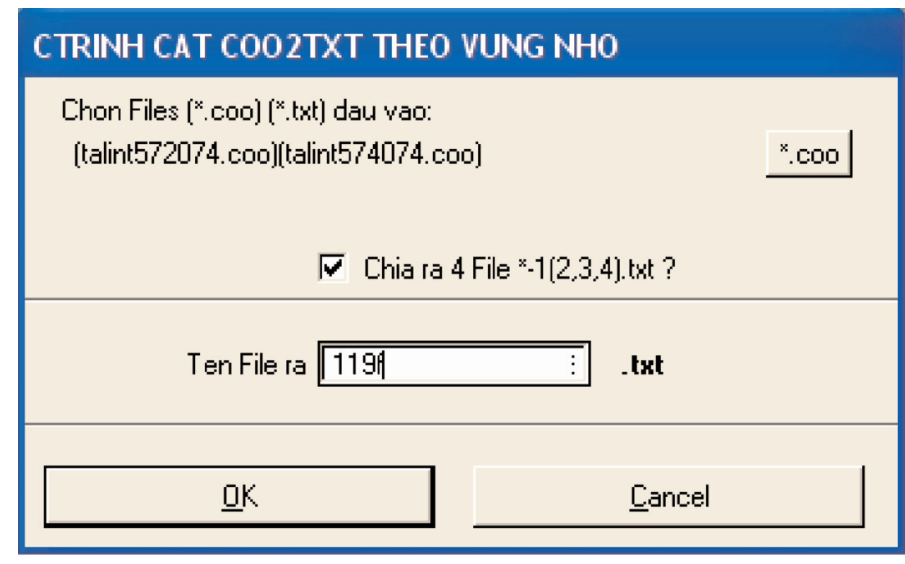

Hình 8: Giao diện macro chia file dữ liệu DTM 


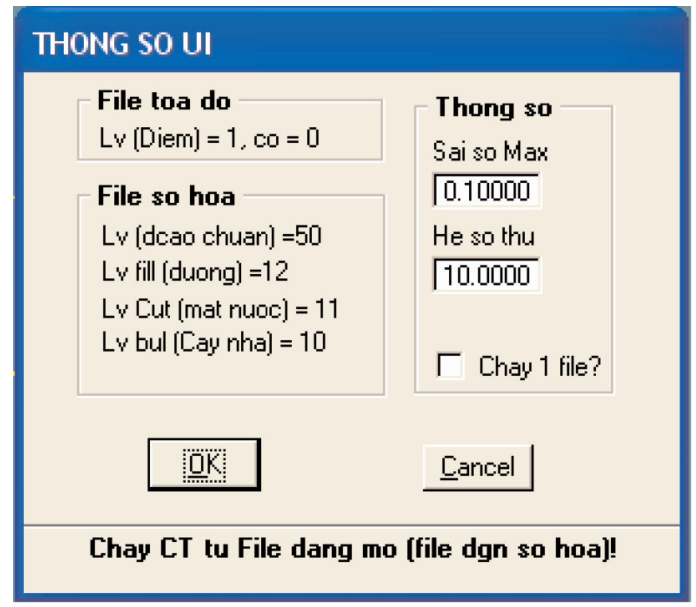

Hình 9: Giao diện macro chuẩn hoá DTM

MicroStation thực hiện xuất file DTM theo định dạng quy định để lưu trữ và giao nộp. (Xem hinh 10)

\section{Kết luận}

Các Module phần mềm trên đã được áp dụng trong ước tính thông số thiết kế, thực hiện bay quét, quản lý và xử lý dữ liệu LiDAR - ảnh số trong các dự án sản xuất của Tổng công ty Tài nguyên và Môi trường Việt Nam. Kết quả thực tế đã chứng minh hiệu quả sử dụng của các Module này trong hỗ trợ ứng dụng công nghệ LiDAR và máy ảnh số trong thu nhận và xây dựng cơ sở dữ liệu địa lý ở nước ta. $\mathrm{O}$

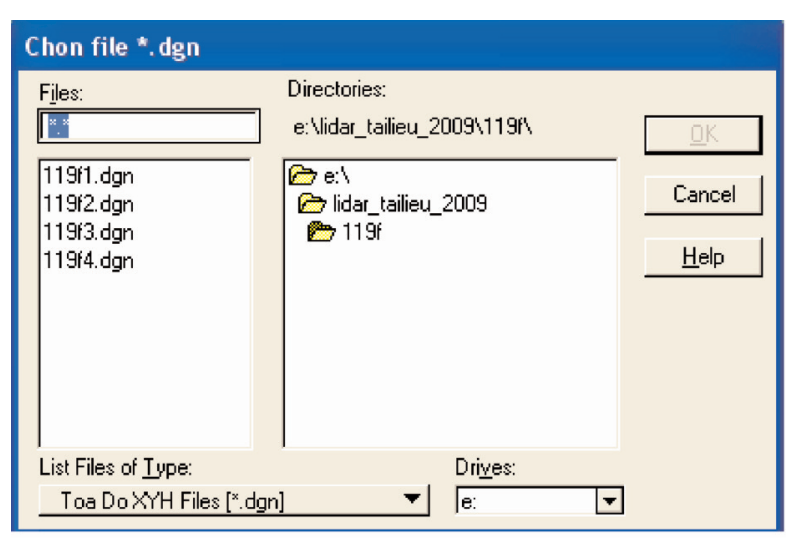

Hình 10: Giao diện macro tự động ghép và xuất file DTM

\section{Tài liệu tham khảo}

[1]. Nguyễn Trường Xuân, Cáp Xuân Tú, 2010. Luận án tiến sỹ kỹ thuật "Nghiên cứu ứng dụng công nghệ tích hợp LiDAR và Máy ảnh số trong thành lập cơ sở dữ liệu mô hình số độ cao và bình đồ trực ảnh tại Việt Nam".

[2]. Toposys, Manual, 2008. Harrier56_LiDAR_Data_Processing. Toposys $\mathrm{GmbH}$, Germany.

[3]. Uwe Lohr, Toposys GmbH, 2003. Precision Lidar Data and True-Ortho Images. Map ASIA. O

\section{Summary}

Development some modules for supporting in integrated LiDAR - Digital camera system in order to create Digital Elevation Model and True-Ortho Images.

Assoc. Prof. Dr. Nguyen Truong Xuan

\section{Dr. Cap Xuan Tu}

Eng. Mai Van Sy

In this paper, we introduce some modules which are builted for supporting technical design and information processing in integrated LiDAR - Digital camera system in order to create Digital Elevation Model and True-Ortho Images in Viet Nam. O

\section{Ngày nhận bài: 20/5/2013.}

